\title{
System design of the physical layer for Loon's high-altitude platform
}

\author{
Sharath Ananth* (D), Ben Wojtowicz, Alfred Cohen, Nidhi Gulia, Arunoday Bhattacharya and Brian Fox
}

\begin{abstract}
This paper describes several aspects of the physical layer and over the air interface of Loon. Loon utilizes stratospheric balloon-based high-altitude platforms (HAPs) that use Long-Term Evolution (LTE) to connect people with standard User Equipment (UEs) to the Internet. In particular, topics covered include the Loon prototype eNodeB (eNB) antenna pattern, the observed channel, UE battery life, and coexistence with terrestrial networks using the same spectrum. While channel models from a HAP to the ground have been well studied in the past, the use of polarization diversity to establish Multi-Input Multi-Output (MIMO) communication to real UEs below $1 \mathrm{GHz}$ has not. In addition, a theoretical analysis of terrestrial coexistence and an analysis of the estimated impact on UE battery life when communicating with HAPs are presented. Finally, results from several measurement campaigns and from experiments with polarization diversity are presented as a spot check of theory.
\end{abstract}

Keywords: Loon, Unmanned areal vehicle (UAV), Channel model, Air-to-ground communication environments, Polarization diversity, Cochannel interference, LTE, 4G, eNB, HAP/HAPS, Cross polar discrimination (XPD), MIMO, HAPS terrestrial overlay

\section{Introduction}

The use of HAPs to deliver Internet connectivity on the ground has been discussed for almost two decades, with many research articles on various aspects of such a system, including the wireless link. Loon has developed the only stratospheric HAP that has provided connectivity to hundreds of thousands of people. To accomplish this, the Loon HAP is comprised of high-altitude balloons, floating between 17 and $21 \mathrm{~km}$ above the Earth, which beam standard compliant LTE signals to the ground. This is accomplished via an eNB that resides on the balloon which facilitates communication with standard UEs. For backhaul, the balloons are able to communicate over multiple balloon-to-balloon hops before landing the traffic on the ground with a balloon-to-ground hop, all using a proprietary high speed link. In this sense, one can consider the balloons as having formed a mesh network in the sky.

The design of such a system needs to address some very interesting challenges:

- Interoperability with regular UEs, including those that are 3GPP Release-8 compliant. Modifications to

*Correspondence: sananth@loon.com

Loon LLC, 100 Mayfield Ave, Mountain View, CA 94043 USA the standard are not desired, as the goal of Loon is to serve users in emerging markets.

- UE antennas are optimized for communication with terrestrial cellular towers and not HAPs.

- Power budget is constrained due to the use of solar power, and mass budget is constrained by the physical limits of the prototype Loon HAP.

- Transmit power on the UE is limited and cannot be increased.

- The spectrum used by the Loon HAP may be the same as that used on the ground by terrestrial cellular operators. Therefore, the Loon HAP needs to coexist with these transmissions.

In prior work, channel models for HAP communications have been researched extensively (e.g., [1-10]). In addition, chapter 10 in [11] goes over recent work in stratospheric channel models. However, only a few papers (e.g., [12-14]) discuss HAP-MIMO channel models and only a few $([15,16])$ discuss the use of polarization to achieve diversity. For instance, [13] discusses the advantages of having MIMO for HAP communication by evaluating the performance of downlink (DL) HAP channels. Through the use of simulation, this work shows that 
using $2 \times 1$ MIMO improves LTE performance by 1.4 to $12.3 \mathrm{~dB}$ and that using $2 \times 2$ MIMO can improve the performance by 7.7 to $15.7 \mathrm{~dB}$. This research uses data from [17] to model the channel as an elevationdependent Ricean. The authors assume two independent, Rician faded channels in their simulation but it is unclear how this independence is achieved. Michailidis and Kanatas [12] derive a three-dimensional, geometrybased, single bounce channel model for MIMO channels in Ricean fading environments. Using derived theoretical expressions, an evaluation of the HAP antenna interelement spacing requirement for achieving uncorrelated responses in HAP MIMO channels is derived. However, using polarization to achieve the uncorrelated channels is not considered. In addition, [9] measures building penetration loss (BPL) as a function of elevation angle and polarization. In order to simulate receive antennas with directional patterns, the authors place antennas in an orthogonal configuration (i.e., one antenna pointing vertically and another pointing horizontally). This configuration is used to measure the impact of polarization on BPL as a function of elevation. Figure 5 from [9] shows a peak differential in BPL of $5 \mathrm{~dB}$ between vertical and horizontal polarization at $2 \mathrm{GHz}$ with test receivers. However, typical UEs do not have nicely orthogonal antenna patterns below $1 \mathrm{GHz}$. Oestges [10] discusses the effect of rain and ice depolarization on a HAP at $47 \mathrm{GHz}$, using dual-polarized antenna arrays. Dong et al. [15] analyzes diversity performance from multiple HAP networks while also considering the single HAP use case. The authors show that due to the very close distance between the antennas in a single HAP, the use of traditional MIMO techniques cannot overcome large-scale fading. Due to the predominant line of sight (LOS) channel conditions in a HAP operating environment, propagation channels are highly correlated and most diversity techniques are not applicable. However, the authors also show that there may be exceptions such as using spatial diversity on the ground or using multiple HAPs. Also, using polarization to achieve diversity from HAPs is not explored. Michailidis et al. [18] provides a mathematical model for polarization-based diversity from HAPs by calculating the XPD between orthogonal polarizations. Figure 2 from this paper is particularly interesting, as the computations show that XPD is expected to be low for an urban region (e.g., London) even when the HAP is directly overhead. This is somewhat counter intuitive, as a high XPD would be expected due to the strong LOS conditions. However, the analysis uses a Ricean $\mathrm{K}$ factor of 0 since it is for a dense urban area. This figure is not applicable to more rural areas, where the Ricean $\mathrm{K}$ factor is high. In addition, the authors assume an isotropic antenna pattern for the UE, which does not hold for typical UEs in real use. Nikolaidis et al. [16] provide measurement data for XPD in LOS channels from airships using dual polarized antennas. Table 1 in this paper shows that an XPD of greater than $15 \mathrm{~dB}$ is expected for all conditions (e.g., LOS, non-LOS). Section III B, and the discussion around the Demmel condition number, leads to the conclusion that at high elevation angles, a large amount of multiplexing (MIMO) based transmission should be expected. However, using co-located HAP antennas at less than $1 \mathrm{GHz}$ with real UEs, given their antenna limitations, has yet to be explored. Using polarization to provide diversity gain in terrestrial base stations has also been researched extensively (e.g., [19-22]). For instance, [19] demonstrates that polarization provides a means of realizing two independently fading signals with co-located antennas by relying on the ability of scatterers in the channel to depolarize and decorrelate the signals. In addition, [21] demonstrates that polarization diversity is mostly preserved in LOS conditions for terrestrial applications. Finally, coexistence of transmissions from HAPs and terrestrial deployments using the same frequency has been researched extensively (e.g., [23-28]). For instance, [23] discusses coexistence of $3 \mathrm{G}$ in disaster scenarios, where some terrestrial towers are disabled due to an emergency. The terrestrial network is then overlapped by a HAP-based $3 G$ network, and the impact of the HAP network to the terrestrial network is analyzed. Based on the parameters chosen, the authors are able to demonstrate that the simultaneous application of HAP and terrestrial networks impacts the terrestrial signal, particularly in suburban and urban macro cellular areas. Likitthanasate et al. [24] discuss coexistence of WiMax at $5 \mathrm{GHz}$. The authors consider a single HAP with a single terrestrial base station located $10 \mathrm{~km}$ away from, but still within, the HAP coverage area. Based on the parameters chosen, the authors conclude that the HAP and terrestrial base station can coexist with low data rate modulation schemes. To get higher data rates, the authors expect that the UE antenna beamwidth would have to be narrow (e.g., less than $30^{\circ}$ ). A similar idea of exploiting antenna directionality in the UE is explored in [25]. Here, the authors discuss coexistence among a constellation of HAPs, where interference from multiple HAPs is reduced by using a narrow antenna beamwidth at the

Table 1 Conducted power by loading per port (assuming $37 \mathrm{dBm}$ per port at maximum loading) for $5 \mathrm{MHz}$ channel bandwidth

\begin{tabular}{llll}
\hline Num PRBs active & \% PRB loading & $\begin{array}{l}\text { Power delta from } \\
\text { peak in } \mathrm{dB}\end{array}$ & $\begin{array}{l}\text { Conducted } \\
\text { power in } \mathrm{dBm}\end{array}$ \\
\hline 0 & 0 & -10.21 & 26.79 \\
4 & 16 & -6.2 & 30.8 \\
12 & 48 & -2.76 & 34.24 \\
19 & 76 & -1.06 & 35.94 \\
25 & 100 & 0.00 & 37
\end{tabular}


UE. Park et al. and Park et al. [26, 27] discuss coexistence of Code Division Multiple Access (CDMA) in terrestrial and HAP deployments. In this research, the minimum distance between terrestrial CDMA coverage regions and HAP CDMA coverage regions is computed to be between 2.5 and $9 \mathrm{~km}$. However, coexistence for LTE at less than $1 \mathrm{GHz}$, with large HAP coverage, and omni-directional UE antennas has not been explored. While existing research has been done in many areas applicable to the Loon use case, many design challenges require more research. This paper intends to build on the existing research by measuring the impact of polarization diversity using co-located HAP antenna bands below $1 \mathrm{GHz}$, demonstrating why the HAP channel model in this use case is not sufficient to support MIMO communications with standard UEs and discussing LTE coexistence between HAPs and terrestrial cellular deployments for real UEs.

\section{Loon system model}

\subsection{Frequency band}

Loon aims to provide network expansion for telecommunication partners by utilizing their existing spectrum allocations and fully integrating into their existing network. Although any of the existing LTE bands available from a partner could be chosen for use on a Loon system, LTE bands below $1 \mathrm{GHz}$ are preferred as they allow for the widest possible coverage. Due to its wide availability, LTE band 28 (703 to $748 \mathrm{MHz}$ for uplink (UL) and 758 to 803 $\mathrm{MHz}$ for DL) has been made available by some of Loon's terrestrial partners for use in actual Loon deployments and will be used for the analysis in this paper.

\section{2 eNB antenna pattern}

The prototype antenna pattern used by Loon's eNB was designed to facilitate outdoor communication over large geographic areas. To this end, a single Loon HAP is expected to cover a region $\sim 40 \mathrm{~km}$ in radius. Beyond this range, even though a UE can receive Loon's DL signal, the system becomes UL limited. In addition, the prototype antenna pattern was designed to not have peak gain directly below the balloon where path loss is the lowest.

Tables 5, 6, 7, and 8 in [29] point out that a coupling loss of no more than $132 \mathrm{~dB}$ is required to achieve $384 \mathrm{kbps}$ on the UL and a coupling loss of no more than $140 \mathrm{~dB}$ is required to achieve $14.4 \mathrm{kbps}$. Assuming a UE antenna gain, including body loss, of negative $10 \mathrm{~dB}$ on average, the antenna gain required at the eNB can be derived as:

AntennaGain $>=$ PathLoss $+10 \mathrm{~dB}-$ CouplingLoss

Where PathLoss is due to free space path loss (FSPL) and CouplingLoss is either $132 \mathrm{~dB}$ or $140 \mathrm{~dB}$. The actual gain of the prototype antenna for Loon's eNB is plotted against these two coupling losses in Fig. 1. As can be seen,
$384 \mathrm{kbps}$ on the UL is expected up to $\sim 65^{\circ}(\sim 43 \mathrm{~km}$ radius) for an outdoor UE with LOS conditions.

In addition, Loon's eNB uses a two transmit and two receive antenna configuration with co-located antenna elements using two different linear polarizations (horizontal and vertical). Experimentation has been performed with other antenna configurations, including spatial separation.

\subsection{UE antenna pattern}

Research has shown that achieving diversity at the UE for frequencies below $1 \mathrm{GHz}$ is difficult. For example, [30] say that, in general, two linearly polarized antennas, located orthogonal to each other, provide polarization diversity by reducing the mutual coupling. They go on to point out that this technique does not work well for UE antennas at lower frequencies (e.g., LTE at $700 \mathrm{MHz}$ ) due to ground plane sizes being much smaller than wavelength $(\lambda$ is 429 $\mathrm{mm}$ at $700 \mathrm{MHz}$ ). Derneryd et al. [31] show that there is a real challenge when designing multiple antennas with low antenna correlation and high efficiency in small hand-held devices, especially at low frequencies. Hagerman et al. [32] describe the result of a field study, conducted by Ericsson and Verizon, which shows that with careful placement of UE antennas it is possible to achieve good MIMO rank even for LTE at $700 \mathrm{MHz}$. This study used mock UEs with various antenna placements and sizes corresponding to smart phones and feature phones. These UEs were then tested for MIMO rank and throughput in a precommercial LTE network. Given that the feature phones have a smaller size, the observed rank-2 MIMO performance in this case was lower than was observed in smart phones. However, the study did not differentiate between polarization and spatial diversity when assessing MIMO performance. This is key, since spatial diversity is difficult to achieve from HAPs, as will be shown.

A typical metric used in LTE handset antenna design is the Envelope Correlation Coefficient (ECC) as described in [30]. In order to achieve good MIMO performance, an ECC value of less than 0.5 is recommended. Typically, UEs exhibit high ECC for the lower bands and lower ECC for the higher bands. For example, a typical popular smart phone will have an ECC around 0.4 to 0.5 at $700 \mathrm{MHz}$ and an ECC of lower than 0.1 at higher frequencies. However, the ECC metric treats spatial and polarization diversity equally (i.e., we can have low ECC by having two antennas point in the same direction with orthogonal polarization or we can have low ECC by having two antennas pointing in different directions with the same polarization). From inspecting the antenna radiation patterns of popular UEs, low ECC in the $700 \mathrm{MHz}$ range is achieved by the antenna pointing in different directions rather than polarization diversity. An impact analysis of antenna pointing direction on ECC can be performed by computing the ECC using 


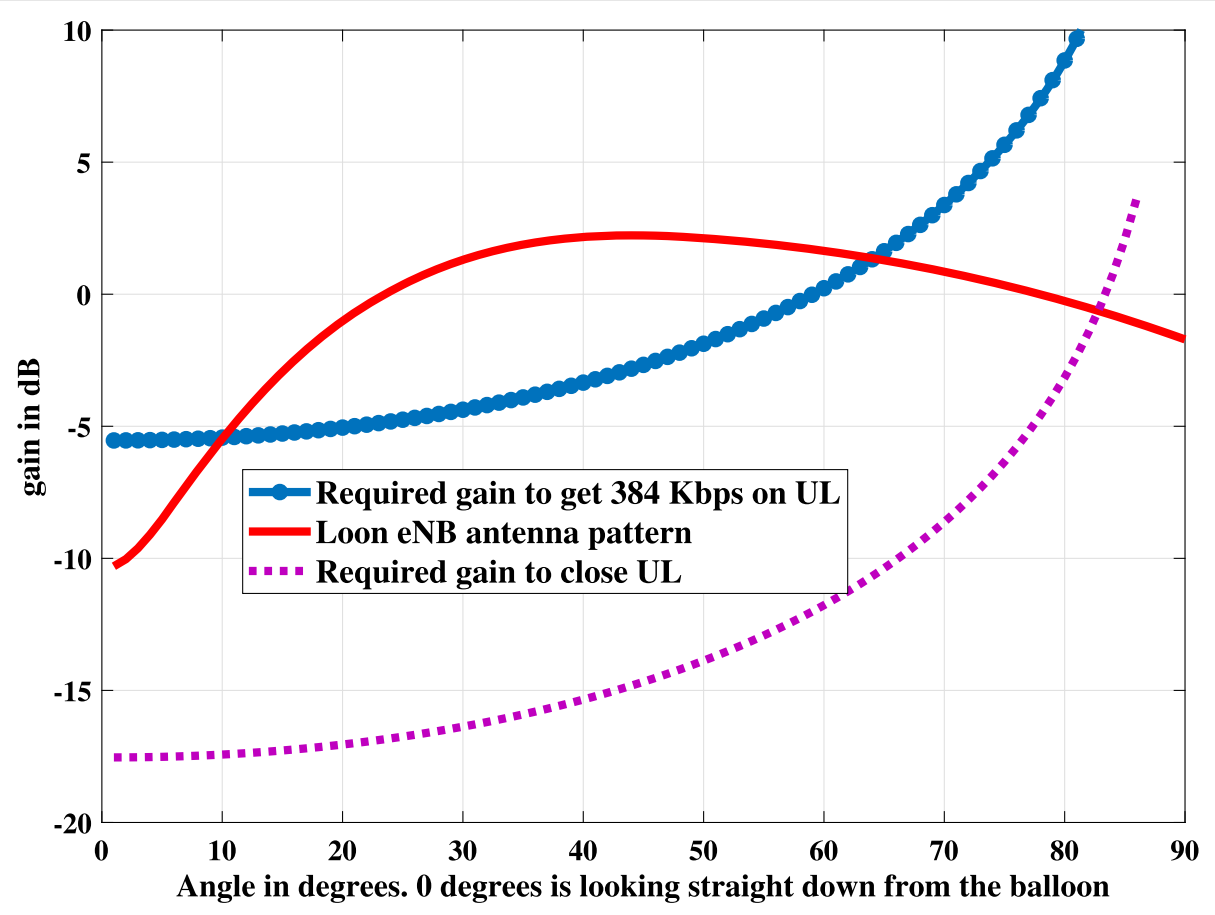

Fig. 1 Loon eNB prototype antenna gain

the total antenna gain, as opposed to the usual computation using the two orthogonal polarizations. If the ECC obtained by these two methods are roughly the same one can conclude that the primary driver for ECC is antenna pointing rather than polarization. If the ECC obtained by the total gain method is high but the ECC obtained by the normal computation method is low, one can conclude that the low ECC is obtained predominantly achieved by polarization diversity. For typical UEs, we observe that modified ECC is only 20 to $30 \%$ higher than the regular ECC computation (at low frequencies). This further corroborates that the low ECC at $700 \mathrm{MHz}$ is due primarily to the antennas pointing in different directions.

Figure 2 shows the total directivity, phi-polarized directivity, and theta-polarized directivity of an a typical popular smart phone. From these plots, it can be seen that the main and diversity antenna patterns point in different directions and that the theta-polarized directivity of both antennas is quite poor. This leads to the UE having predominantly one polarization requiring very strong signal strengths to overcome the weak gain for the second polarization to allow MIMO communication. However, in this scenario, there would be an imbalance in the channel capacity between the two MIMO streams due to the large gain difference.

\section{4 eNB transmit power}

The Loon eNB is currently configured for $37 \mathrm{dBm}$ of conducted power per transmit port, giving a total conducted power of $40 \mathrm{dBm}$ across both ports. The peak conducted power is only achieved when the eNB has 100\% Physical Resource Block (PRB) utilization and is reduced as the number of utilized PRBs is reduced. This property is shown in Table 1 for a $5-\mathrm{MHz}$ channel bandwidth.

In addition, signal strength from a terrestrial network will dominate at the UE as a Loon HAP approaches a more dense deployment. When accounting for proper reselection, UEs will prefer camping on a terrestrial tower compared to the Loon HAP. Due to the lowered demand, the transmitted peak power from the Loon HAP is also reduced.

\subsection{Channel model}

Due to the coverage pattern from a single Loon HAP being $\sim 40 \mathrm{~km}$ and the relatively low rate of drift, the channel for most outdoor UEs is expected to be LOS Additive White Gaussian Noise (AWGN). Shimamoto et al. [17] describe the standard deviation in mean received power versus elevation angle from a HAP. From this, a standard deviation of $0.5 \mathrm{~dB}$ (a Ricean $\mathrm{K}$ factor of around 18) is expected at high elevation angles (i.e., when a HAP is directly overhead), a standard deviation of $3.9 \mathrm{~dB}$ is expected at an elevation angle of $40^{\circ}$, and a standard deviation of $5 \mathrm{~dB}$ (a Ricean $\mathrm{K}$ of 1 ) is expected at low elevation angles of $10^{\circ}$. Although future Loon HAPs will support indoor usage, the prototype Loon system that was used for the measurement campaign in this paper is designed to support 

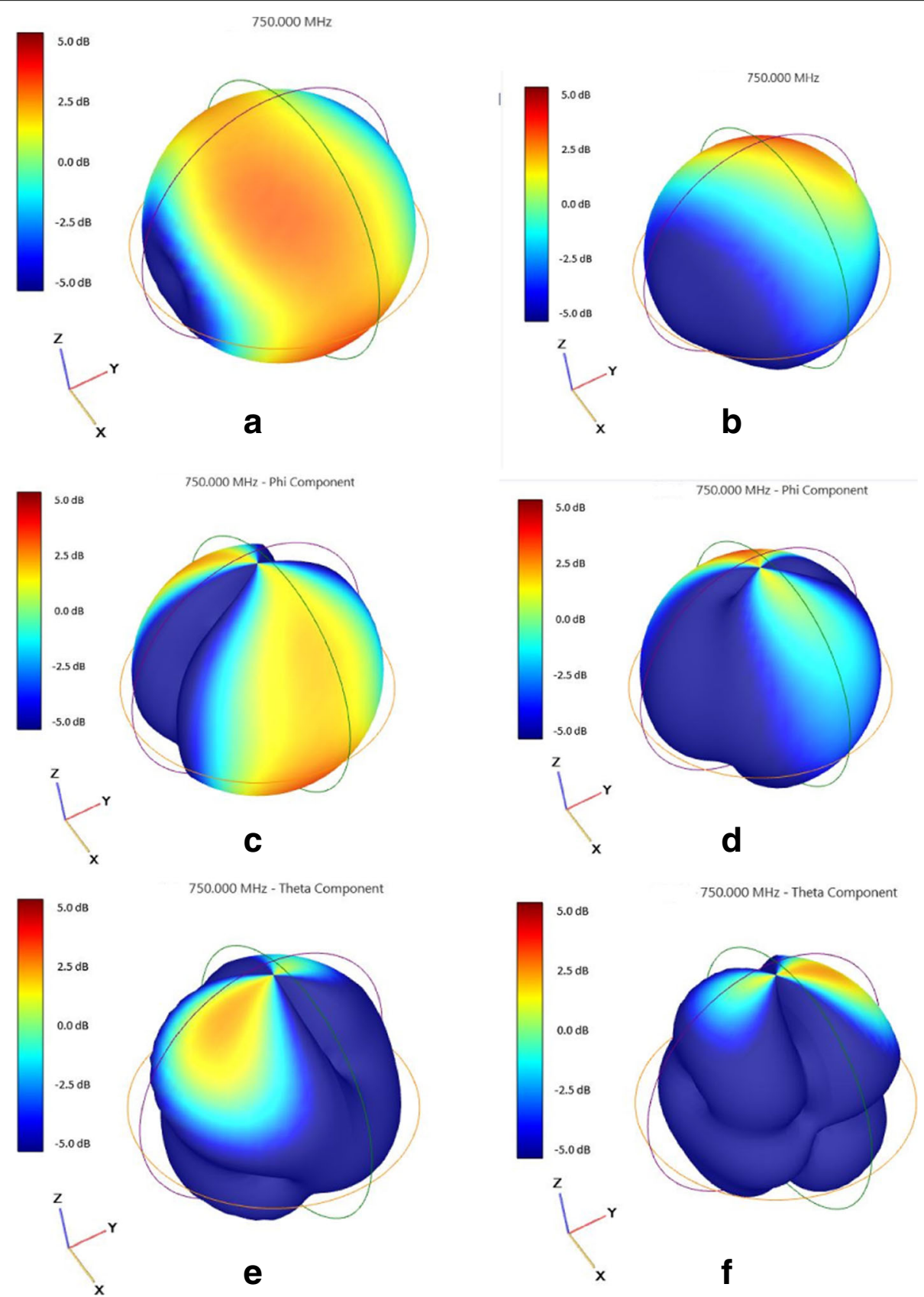

Fig. 2 Typical popular smart phone antenna directivity plots: a total directivity of main antenna, $\mathbf{b}$ total directivity of diversity antenna, $\mathbf{c}$ phi-polarized directivity of main antenna, $\mathbf{d}$ phi-polarized directivity of diversity antenna, $\mathbf{e}$ theta-polarized directivity of main antenna, and $\mathbf{f}$ theta-polarized directivity of diversity antenna

outdoor use cases. Considering the indoor use case, the model described in [17] changes and a higher standard deviation is expected.

\subsection{Terrestrial transmit power and antenna pattern}

This is certainly operator dependent; however, for macro sites, a range of 46 to $49 \mathrm{dBm}$ per antenna port with a peak antenna gain of $18 \mathrm{dBi}$ seems to include most use cases.
For the rest of the analysis, the worst case assumption of 46 and $18 \mathrm{dBi}$ will be used.

\subsection{Spacing of terrestrial towers}

This again is operator dependent and is also geography dependent. At $700 \mathrm{MHz}$, Table 6.33 in [33] gives a cell radius of $6.58 \mathrm{~km}$ in rural areas and $1.88 \mathrm{~km}$ for suburban areas. For the rest of the analysis, $13.16 \mathrm{~km}(2 \times 6.58 \mathrm{~km})$ 
spacing will be used for rural and $3.76 \mathrm{~km}(2 \times 1.88 \mathrm{~km})$ spacing will be used for suburban.

\subsection{Location of users}

Although the prototype Loon HAP was designed for outdoor usage and future Loon HAPs are being designed to additionally support indoor usage, both indoor and outdoor users will be considered in the analysis. It is expected that a higher percentage of users will be indoors.

\subsection{Loon propagation model}

As was already discussed, a LOS-AGWN channel is expected. This channel is dominated by FSPL which will be used for this analysis. Using FSPL for interference modeling is optimistic for propagation prediction and presents a worst case for the coexistence analysis. In addition, FSPL is used in many HAP coexistence studies (e.g., $[26-28,34,35])$.

\subsection{Terrestrial propagation model}

For terrestrial propagation, the impact of terrain must be taken into account. A simplified version of COST231 Hata as given in [33] is used for this analysis. In this model, the eNB height is assumed to be $35 \mathrm{~m}$ and the UE height is assumed to be $1 \mathrm{~m}$. Path loss is then calculated as a function of distance $(d)$ between the UE and the tower in $\mathrm{km}$ :

$$
\text { PathLossSuburban }=113.32+34.8 * \log 10(d)
$$

$$
\text { PathLossRural }=100.15+34.8 * \log 10(d)
$$

Log normal fading margin is not applied here, as this is intended to be a worst case analysis. In typical terrestrial deployments, an additional margin of $10 \mathrm{~dB}$ is expected for $\log$ normal fading as given in Table 11 of [36].

\subsection{Building penetration loss}

BPL is very much dependent on the type of construction used for building roofs. From observation, developing countries have a wide variety of roofs. This ranges from roofs which pass radio frequency (RF) signals with minimal attenuation (e.g., thatch) to roofs which add substantial attenuation (e.g., reinforced concrete). For this analysis, an average of $13 \mathrm{~dB}$ BPL is used when considering the indoor scenario. In the outdoor scenario, BPL is $0 \mathrm{~dB}$.

\subsection{UE characteristics}

As was discussed previously, UE antenna gain and body loss is expected to be around negative $10 \mathrm{~dB}$. However, most studies (e.g., [23, 36]) model the UE antenna gain to be $0 \mathrm{~dB}$. Considering that there may be customer- premises equipments (CPEs) with higher antenna gain, coexistence analysis in this paper will use $0 \mathrm{~dB}$.

\subsection{Minimum required signal to interference and noise ratio (SINR), maximum possible SINR, and coverage metric}

Table 6.11 from [33] defines $-4 \mathrm{~dB}$ as the minimum required SINR for LTE service, and [37] requires that the error vector magnitude (EVM) for a transmitted signal be less than $8 \%$. This $8 \%$ EVM requirement translates to a peak SINR of $25 \mathrm{~dB}$. For this analysis, the maximum possible SINR is limited to $30 \mathrm{~dB}$ to allow for implementations that outperform the minimum requirement. In addition, [24] defines two metrics called "percentage coverage area served" and "percentage coverage area not served." Using these definitions, this analysis defines a coverage area and measures the SINR distribution. This SINR distribution is measured by sampling the coverage area uniformly and plotting a cumulative distribution function (CDF).

\section{Simulation results and discussion}

\subsection{DL interference}

\subsubsection{Simple interference without Loon HAPs}

Considering first a single terrestrial tower without a Loon HAP, Fig. 3 shows the expected SINR on the ground. When a second terrestrial tower is added $13.16 \mathrm{~km}$ from the original tower at the same frequency (i.e. a rural, single frequency network), Fig. 4 shows the expected SINR from both towers combined. Although the plots show that the cell radius extends out to $20 \mathrm{~km}$, this is only due to the optimistic assumptions on UE antenna gain $(0 \mathrm{~dB})$ and lack of log normal fading margin. As described earlier, this is to present a worst case scenario. In all cases, the SINR is limited to $30 \mathrm{~dB}$. As can be seen, the presence of additional terrestrial towers reduces the peak SINR at the UE from greater than $30 \mathrm{~dB}$ to slightly less than $15 \mathrm{~dB}$.

\subsubsection{Simple interference with a Loon HAP}

From this base analysis, a Loon HAP with 100\% eNB load is added at a distance of $30 \mathrm{~km}$ from the terrestrial tower. This distance aligns the peak antenna gain from the Loon HAP with the terrestrial tower. In order to represent the worst case, all UEs are considered to be outdoors and the Loon HAP is at maximum transmit power.

Figure 5 shows the expected SINR for this case. As expected, there is a further drop in the SINR near the terrestrial towers. However, there is also a large region where coverage has improved substantially.

Having considered the worst case scenario for coexistence analysis with all UEs outdoors, it is interesting to consider the inverse condition with all UEs indoors. 


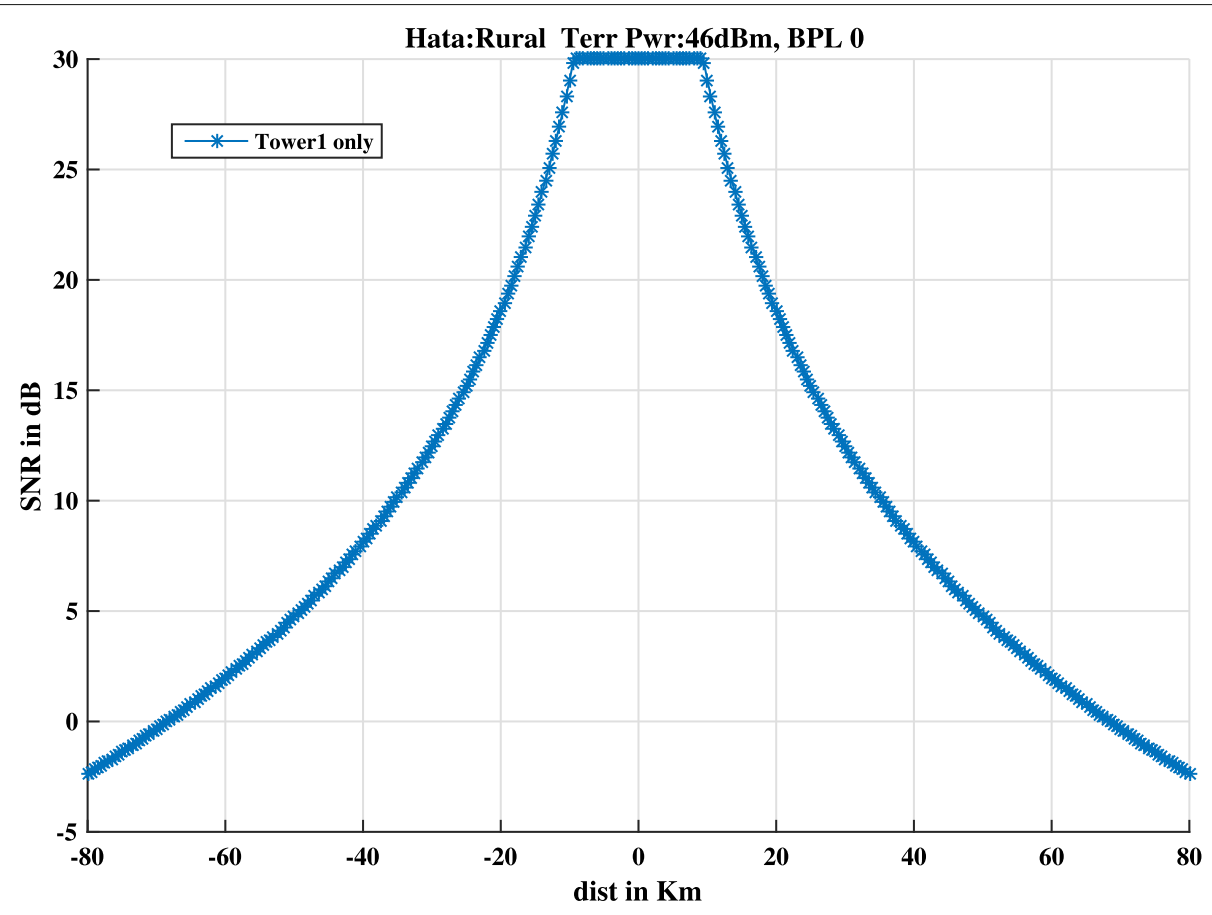

Fig. 3 Expected outdoor SINR with a single rural terrestrial tower

The SINR from this scenario is shown in Fig. 6. In this case, there is minimal impact due to the Loon HAP while the outdoor coverage gain is still realized.

Considering now a suburban case, Fig. 7 shows the two terrestrial towers spaced $3.76 \mathrm{~km}$ apart for outdoor UEs, still with $100 \%$ load on the Loon eNB.
In this case, there is a degradation in SINR due to the Loon HAP. However, it is substantially less than the degradation in SINR due to the terrestrial towers interfering with each other.

In all cases and in all areas, the combined curves are above the minimum SINR threshold of $-4 \mathrm{~dB}$.

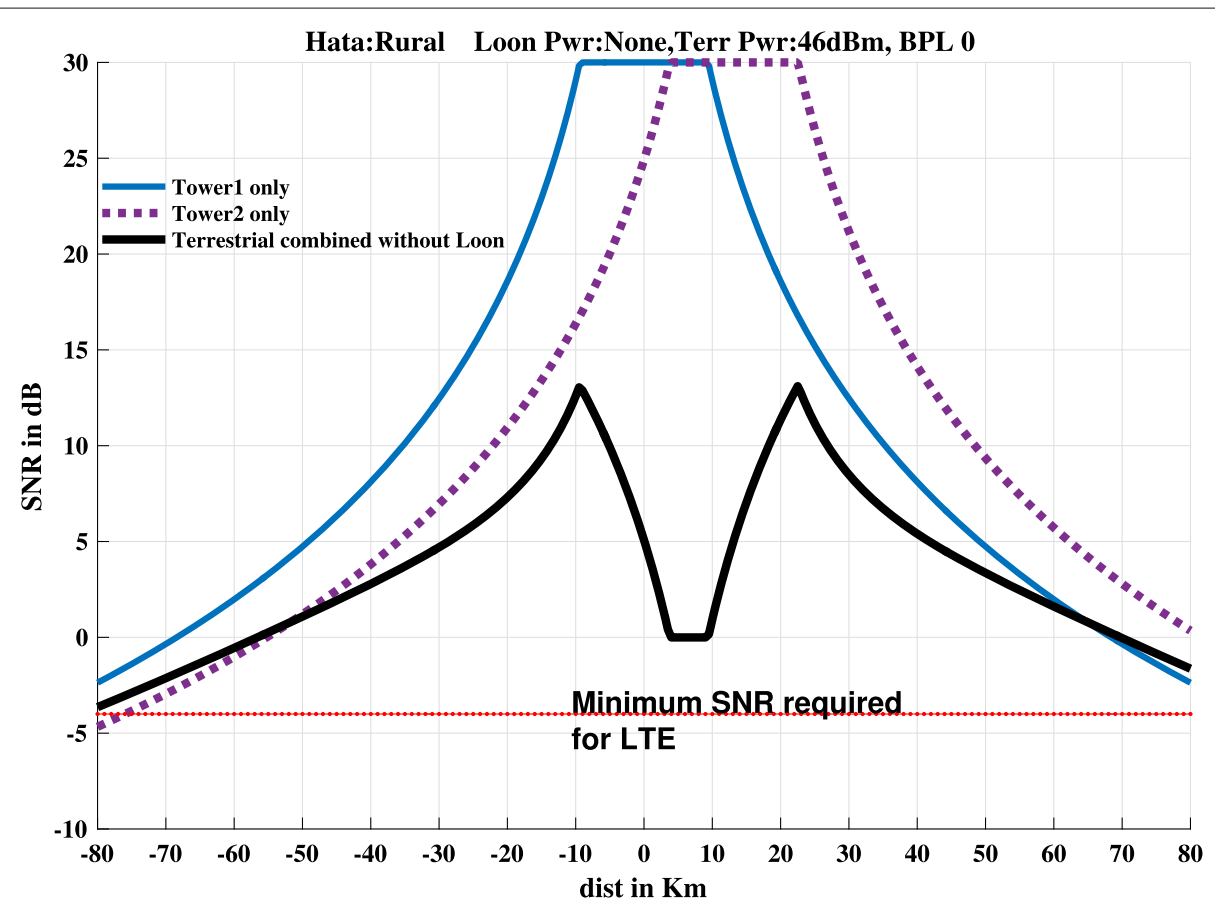

Fig. 4 Expected outdoor SINR with two rural terrestrial towers 


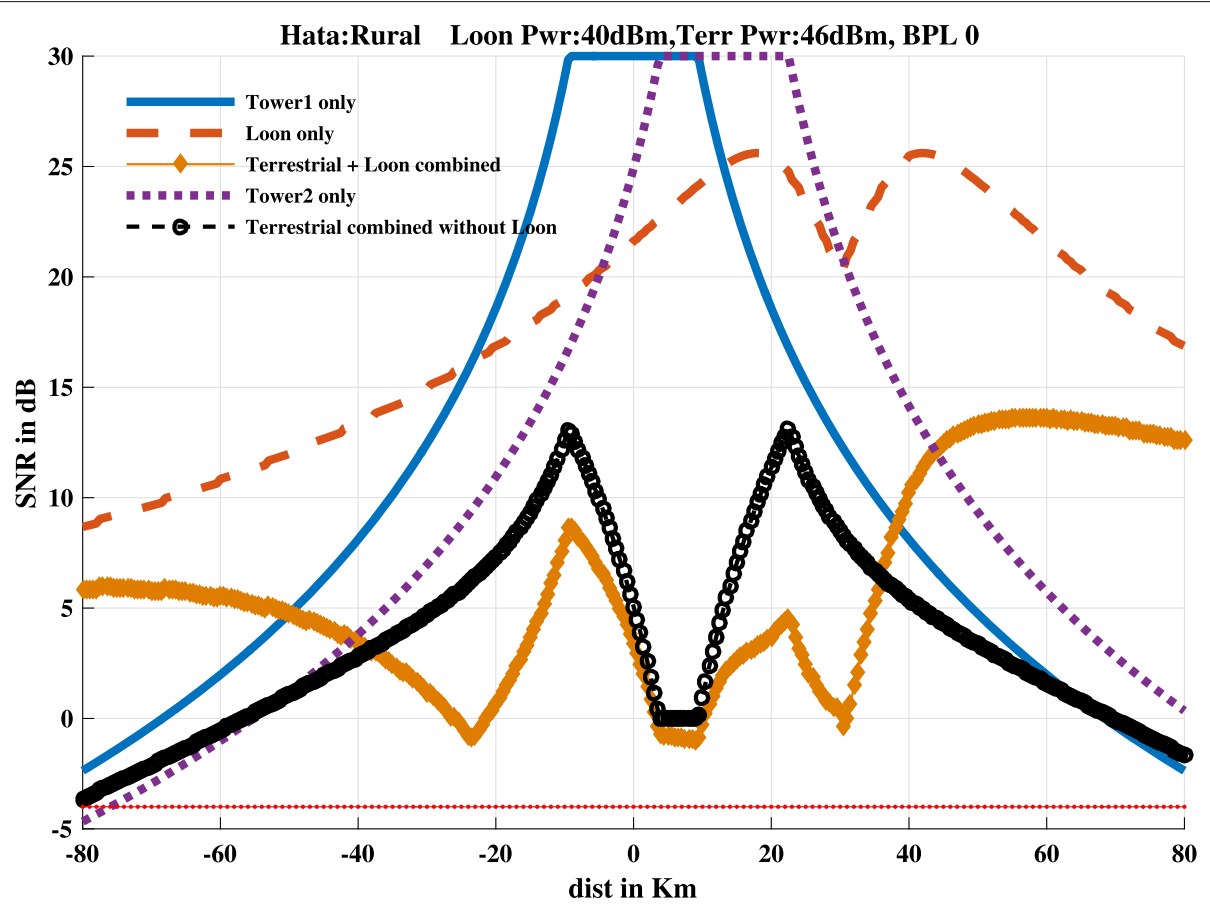

Fig. 5 Expected outdoor SINR with two rural terrestrial towers and a Loon HAP

This means that even though the interference causes cell shrinkage, with proper re-selection, UEs do not lose coverage in any locations and there is a substantial increase in coverage due to the presence of the Loon HAP.

\subsubsection{Interference with terrestrial deployment}

Moving beyond the simplified terrestrial case of two towers, a terrestrial deployment with 37 hexagonal sectors is considered. The radius of each cell is $6.58 \mathrm{~km}$ to simulate a rural deployment. All users are considered to be

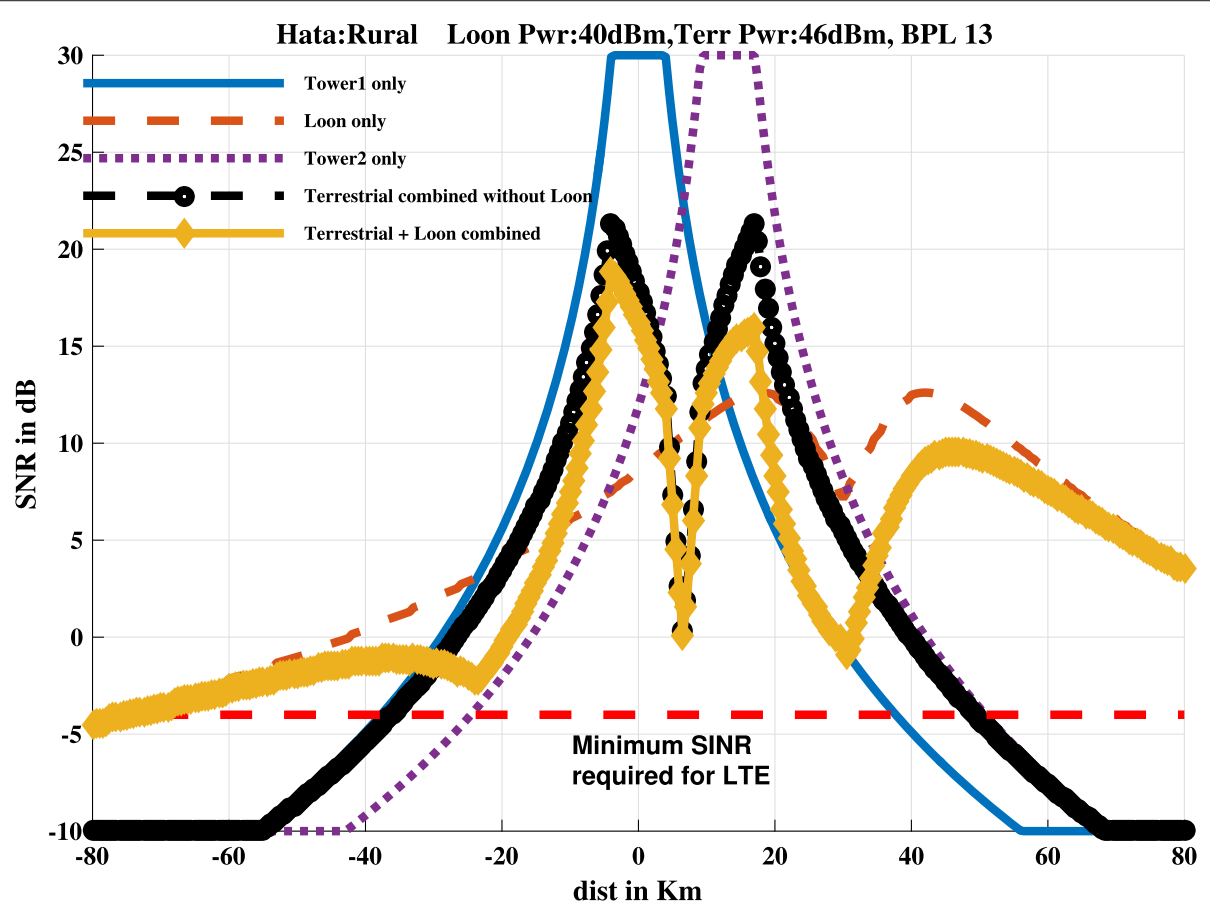

Fig. 6 Expected indoor SINR with two rural terrestrial towers and a Loon HAP 


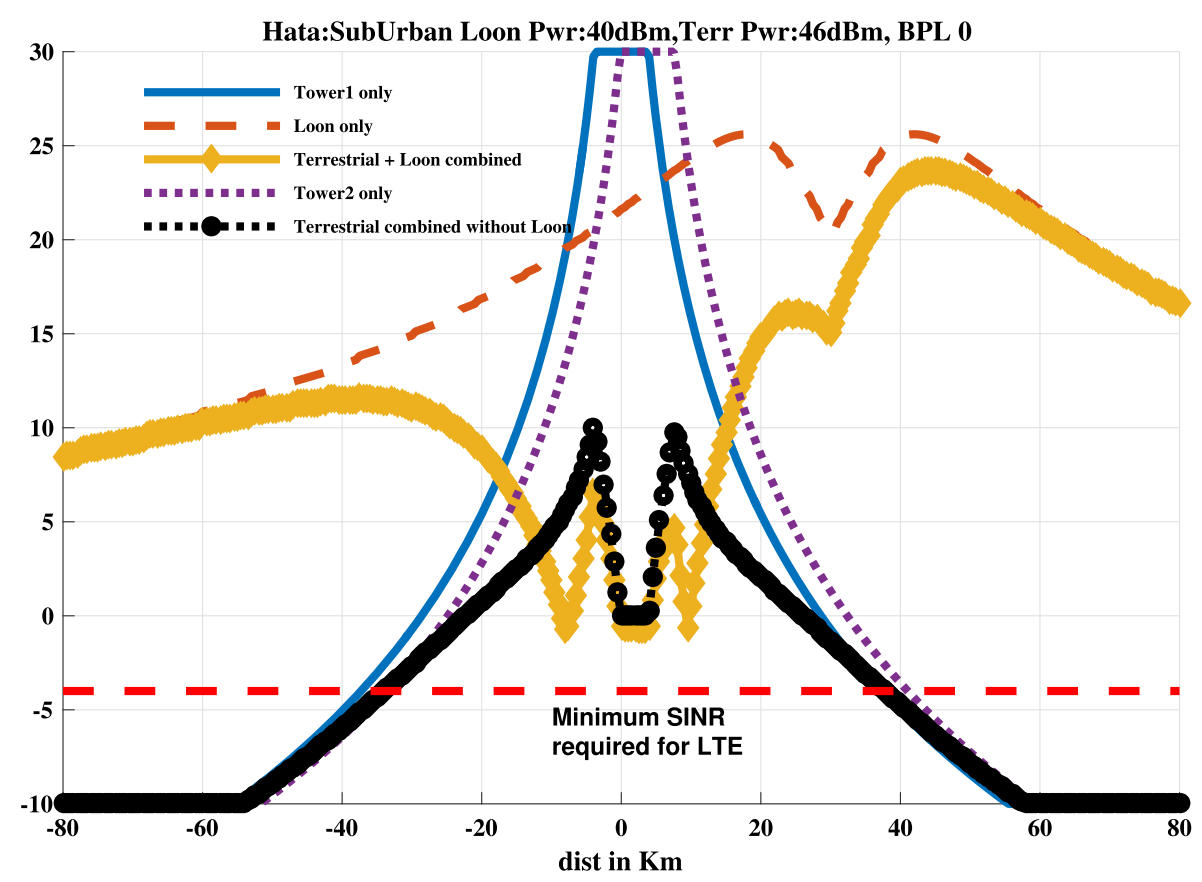

Fig. 7 Expected outdoor SINR with two suburban terrestrial towers and a Loon HAP

outdoors to simulate the worst case. This deployment scenario results in a coverage area with $46 \mathrm{~km}$ radius, the SINR on the ground for this deployment can be seen in Fig. 8.

For the analysis, a single Loon HAP is placed randomly within the $46 \mathrm{~km}$. In addition, the Loon eNB is assumed to have $100 \%$ load for a worst case analysis and toroidal wrap is used to model a wider area.

Figure 9 shows the resulting CDF of the SINR for the entire region, computed before and after placement of the Loon HAP. As can be seen, a negligible delta is observed when a Loon HAP is directly over a fully built out terrestrial network.

In order to model situations where the terrestrial network is not fully built out, the same basic model can be used with just 3 terrestrial towers instead of 37 . Figure 10 shows three different CDFs of SINR from this scenario.

The first CDF shows the SINR of the terrestrial network without the Loon HAP. The second CDF shows the SINR of the network when the Loon HAP is added. As can be seen, a large delta is observed in SINR and the degradation of the terrestrial network is non-negligible when the Loon HAP is present. The third CDF shows the SINR of the terrestrial with a Loon HAP added assuming that reselection is happening at the UE without any hysteresis. In this case, the UE will always choose the strongest signal, while all other signals contribute as interference. Under these assumptions, there is no degradation of SINR on average, since a decrease in SINR is seen above $\sim 6 \mathrm{~dB}$ due to interference and an increase is seen below due to the large coverage improvement from the Loon HAP. This is intuitive as there are large coverage holes that are covered by the Loon HAP when there are only 3 terrestrial towers.

\subsection{UL interference}

For the uplink, Loon's eNB will naturally observe signals from UEs within $\sim 40 \mathrm{~km}$ that are communicating with terrestrial towers. In fact, there is a direct link between the number of terrestrial towers the loading experienced in these terrestrial towers, the roof material in these locations and the amount of UL interference that is to be expected. However, in general and with proper reselection, UEs will prefer a terrestrial network if one is available. As a Loon HAP approaches a large terrestrial deployment, this means that fewer UEs will stay camped on the Loon HAP. This certainly reduces the amount of DL interference which the Loon HAP generates, but it also allows for better frequency selective scheduling in the UL for the remaining UEs.

\subsection{UE transmit power and battery life}

Due to the larger distance between a Loon HAP and UEs, as compared to a terrestrial use case, a common assumption is that the UE transmits at higher power more often, leading to a reduction in battery life. This can be analyzed by first understanding the distribution of UE transmit powers in terrestrial networks. Joshi et al. [38] shows that in current terrestrial networks a UE is 

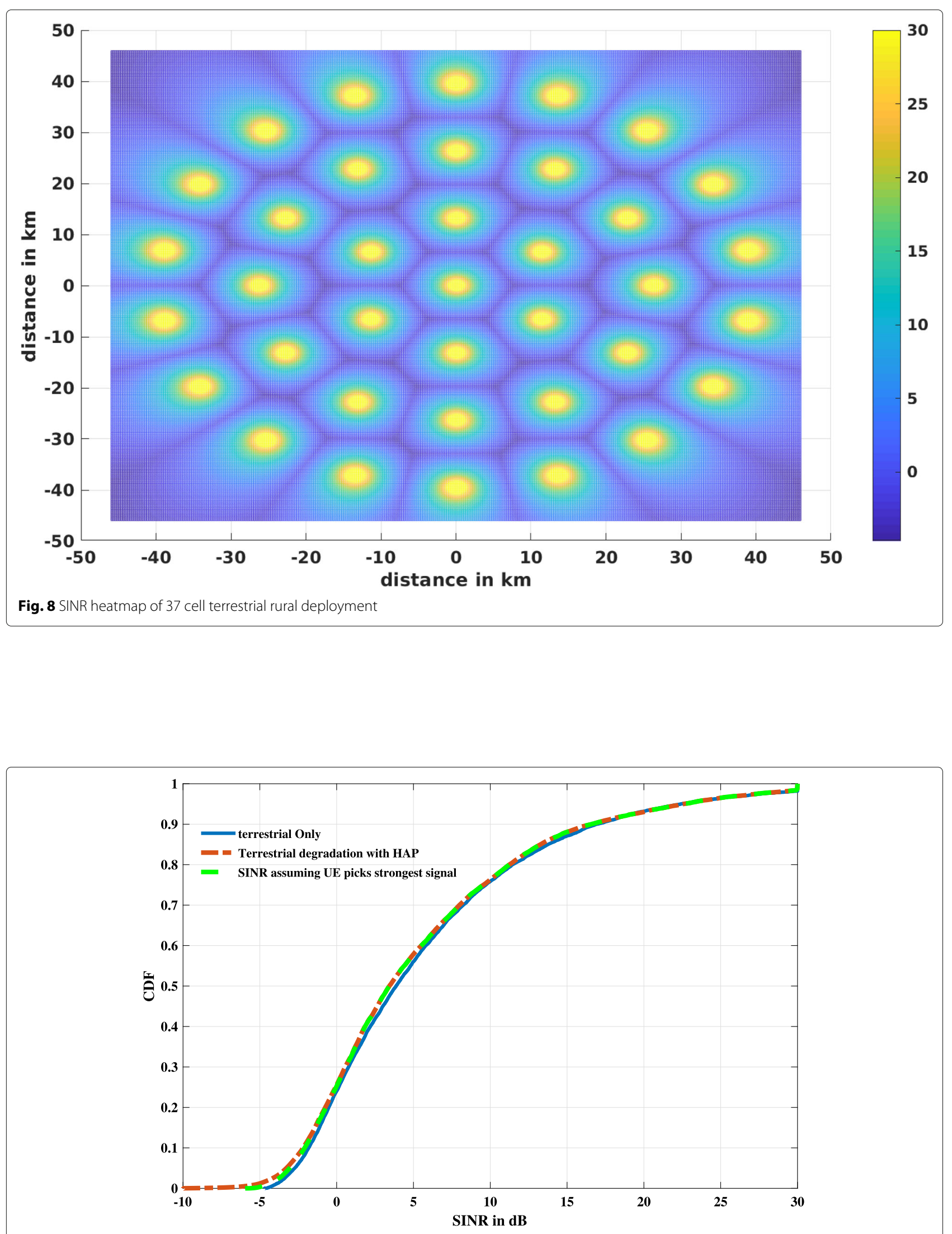

Fig. 9 CDF of SINR before and after placement of a Loon HAP over a 37 cell terrestrial rural deployment 


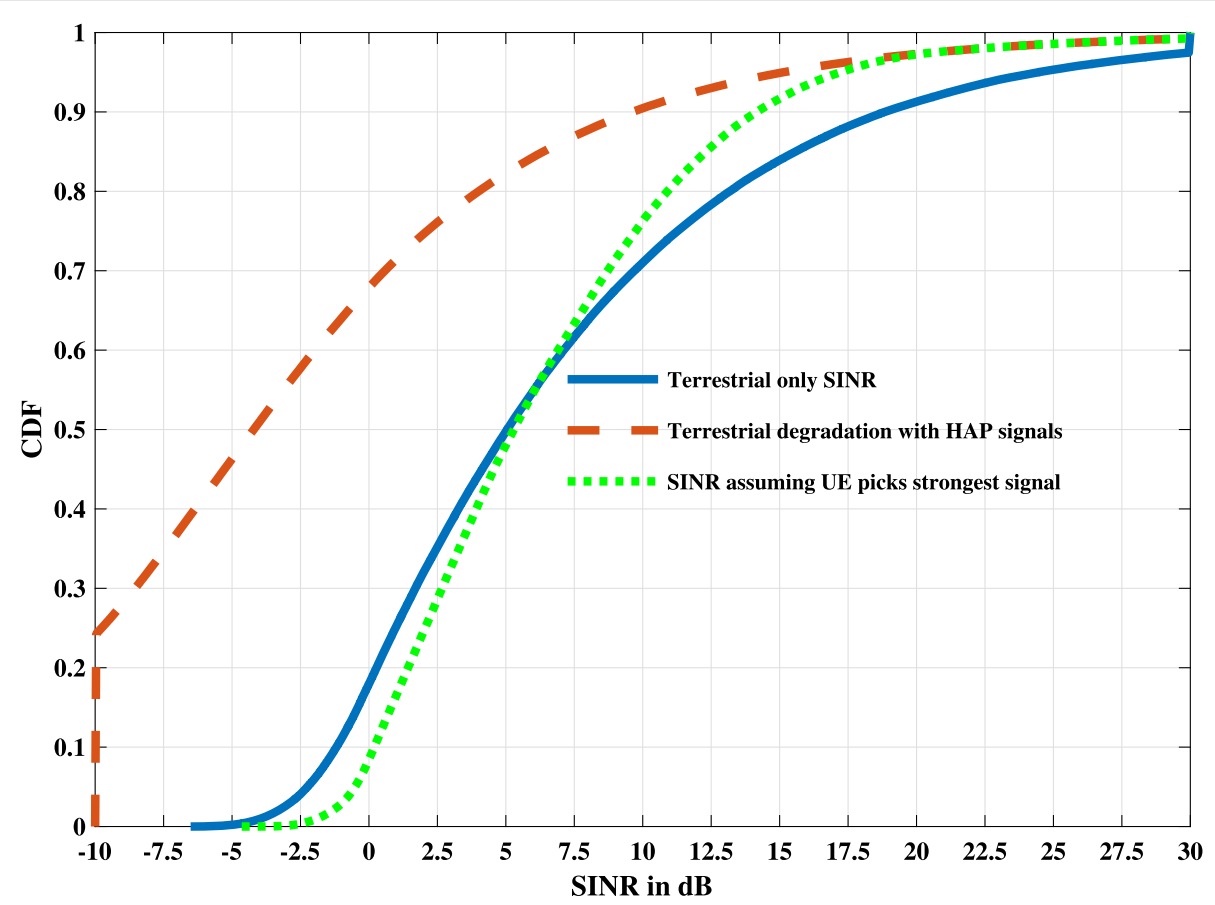

Fig. 10 CDF of SINR before and after placement of a Loon HAP over a 3 cell terrestrial rural deployment

transmitting at maximum power $50 \%$ of the time. In rural areas, this paper also shows that the $95 \%$ case has a UE transmitting $\sim 7.9 \mathrm{~s}$ out of $15 \mathrm{~min}$, while the average is $\sim 3.8$ s out of $15 \mathrm{~min}$. Given this information, the following worst case assumptions will be used for the rest of the analysis:

- Eight seconds of UL transmission out of $15 \mathrm{~min}$

- UL transmissions will be at maximum power $50 \%$ of the time and $12 \mathrm{~dB}$ lower for the remaining time

In addition, Fig. 1 from [39] shows that $2.6 \mathrm{~W}$ are consumed when a UE transmits at maximum power. The same figure shows that $1.7 \mathrm{~W}$ are consumed when transmitting $12 \mathrm{~dB}$ below maximum. Finally, Fig. 3 from [40] shows that the average power consumption during light sleep is $11 \mathrm{~mW}$ per $1 \mathrm{~ms}$ and the average power consumption while receiving is $500 \mathrm{~mW}$ per $1 \mathrm{~ms}$.
Considering a typical paging duty cycle of $640 \mathrm{~ms}$ as shown in Fig. 11, the total energy consumption in idle mode is:

$$
11 \mathrm{~mW} \times 637 \mathrm{~ms}+500 \mathrm{~mW} \times 3 \mathrm{~ms}=8507 \mu \mathrm{J}
$$

Over $14 \mathrm{~min}$ and $52 \mathrm{~s}$, this amounts to:

$$
\frac{(14 \mathrm{~min} \times 60+52 \mathrm{~s}) \times 0.0085 \mathrm{~J}}{0.64 \mathrm{~S}}=11.8 \mathrm{~J}
$$

Total energy consumption for $8 \mathrm{~s}$ of UL transmission is then:

$$
2.6 \mathrm{~W} \times 4 \mathrm{~s}+1.7 \mathrm{~W} \times 4 \mathrm{~s}=17.2 \mathrm{~J}
$$

And total energy consumption over $15 \mathrm{~min}$ is then:

$$
11.8 \mathrm{~J}+17.2 \mathrm{~J}=29 \mathrm{~J}
$$

Considering the worst case for a Loon HAP (i.e., the UE transmit power is always at a maximum), the same math yields:

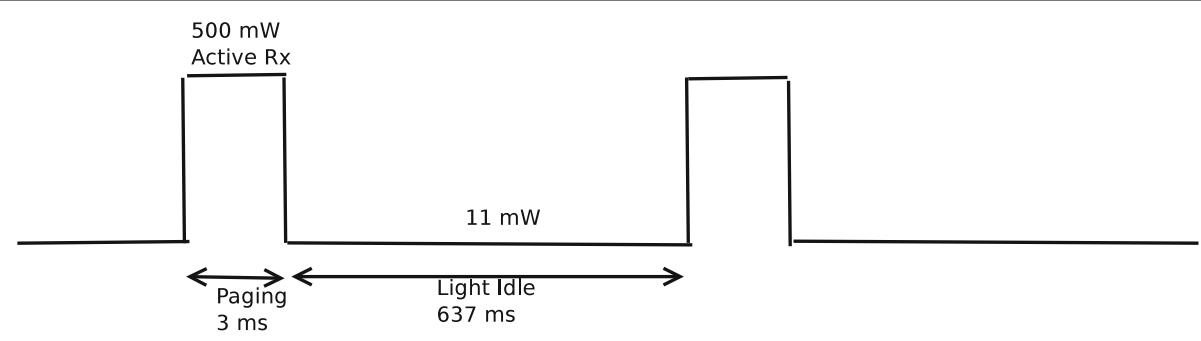

Fig. 11 Modem power profile for a typical paging cycle 


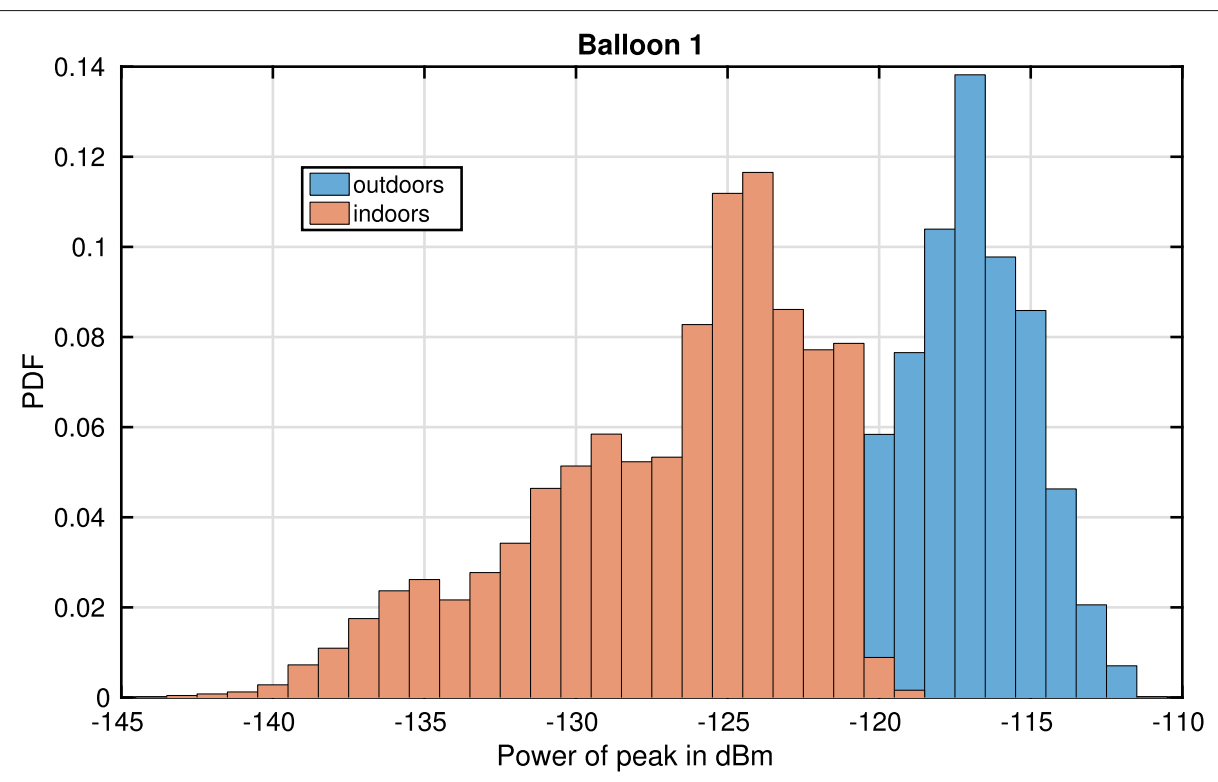

Fig. 12 Signal power distribution from Loon HAP 1 ( $40 \mathrm{~km}$ away), indoor vs outdoor

$$
11.8 \mathrm{~J}+2.6 \mathrm{~W} \times 8 \mathrm{~s}=32.6 \mathrm{~J}
$$

Which represents an increase of approximately $11 \%$ in power consumption for the modem. A low end UE battery is typically $2600 \mathrm{mAH}$ at $3.6 \mathrm{~V}$ and can be assumed to last $24 \mathrm{~h}$ under normal use. So, overall the UE consumes 33,696 J per day or $351 \mathrm{~J}$ per $15 \mathrm{~min}$. Therefore, a delta of $3.6 \mathrm{~J}$ represents an increase of approximately $1 \%$ in UE battery life, which is negligible.

\subsection{Future work}

This analysis was done assuming $100 \%$ frequency reuse between the terrestrial network and the Loon network. However, it may be advantageous for the Loon network to use a smaller channel bandwidth as compared to the terrestrial network. For instance, the Loon network could use $10 \mathrm{MHz}$ when the terrestrial network is using $20 \mathrm{MHz}$. This has the advantage of reducing the overall DL interference on the terrestrial network. In addition, if standard interference coordination techniques (e.g., Inter Cell Interference Coordination (ICIC), enhanced Inter Cell Interference Coordination (eICIC) are used, interference in both the UL and DL can be further mitigated.

\section{Experimental/measurement campaign}

Testing was conducted in both Puerto Rico and Peru during Loon's response to Hurricane Maria (September/October 2017) and the El Niño floods (March/April 2017), respectively. During this testing, Rohde-Schwarz

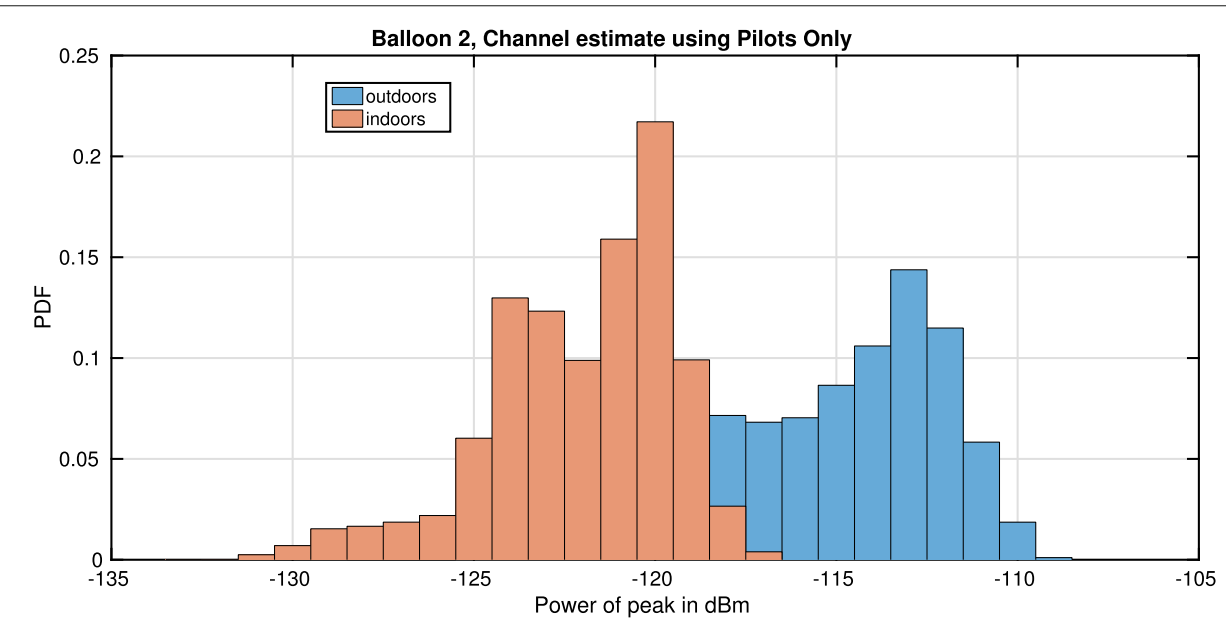

Fig. 13 Signal power distribution from Loon HAP 2 ( $25 \mathrm{~km}$ away), indoor vs outdoor 


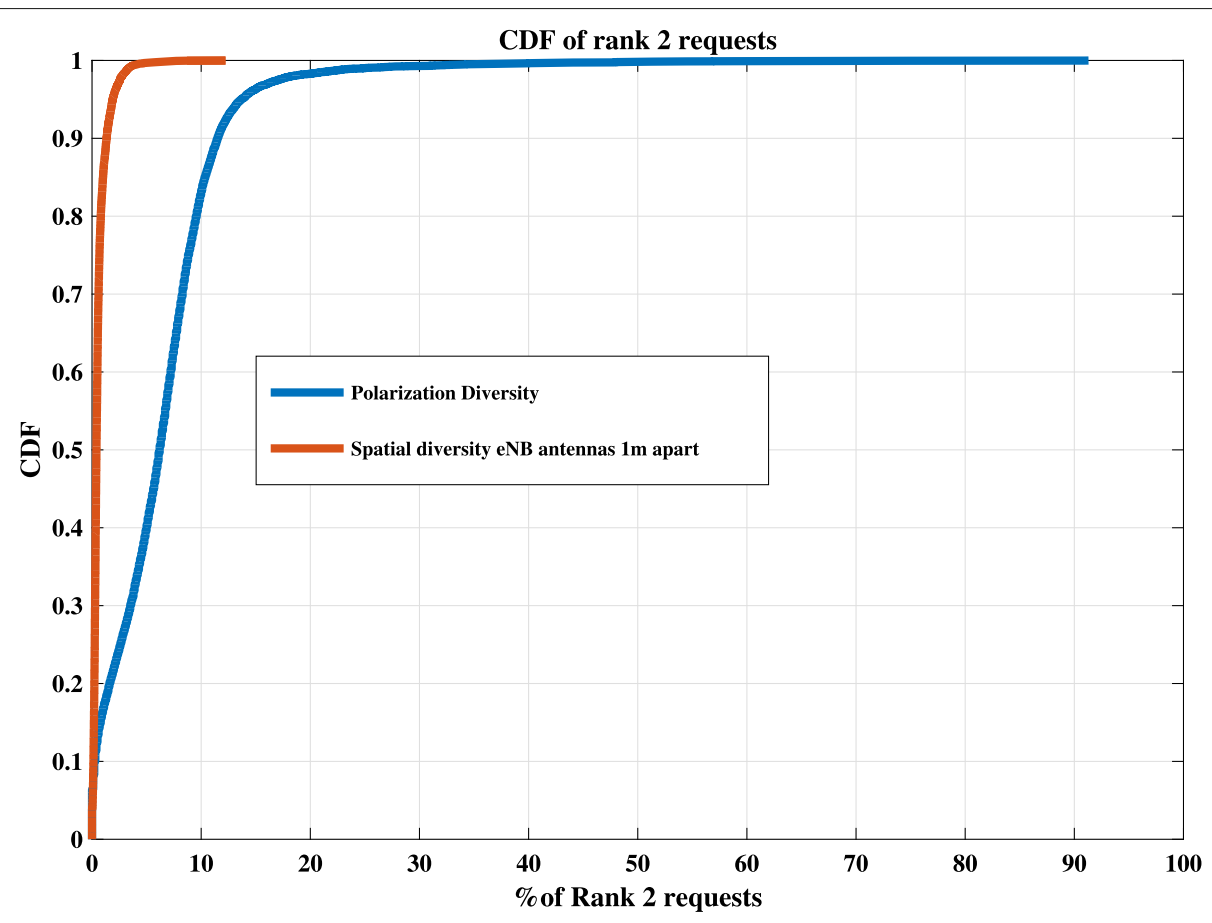

Fig. 14 CDF of percent of rank 2 requests

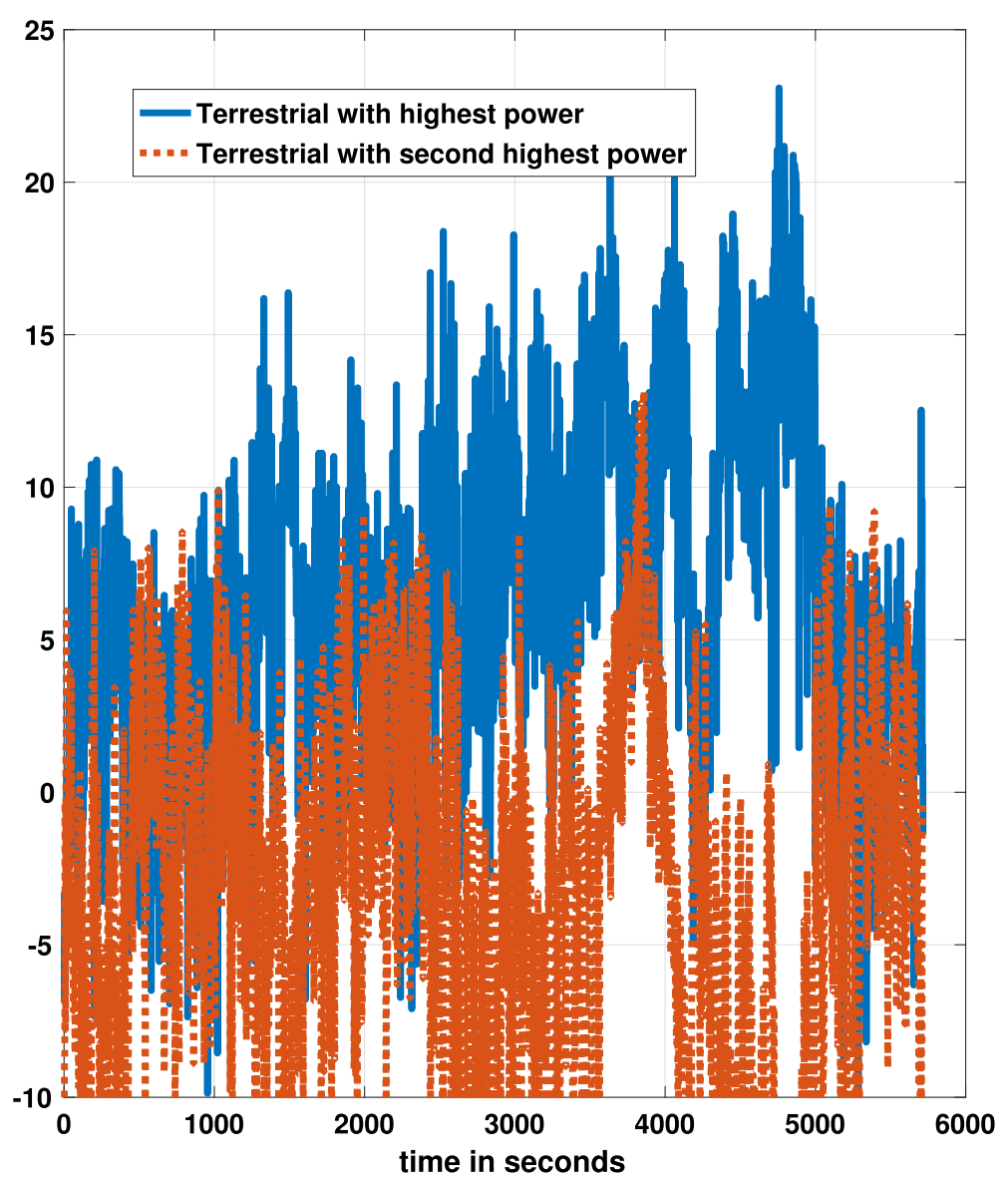

Fig. 15 SINR from two terrestrial towers 
TSME ultra compact drive test equipment [41] was used to measure the channel characteristics while Key Performance Indicators (KPIs) gathered from the Loon network and the terrestrial network were used to assess overall system performance.

\subsection{Loon to UE channel}

\subsubsection{Received signal power at the UE}

In Puerto Rico, data was captured using the TSME in both indoor and outdoor environments. The TSME includes an omni directional external antenna which has an antenna pattern that is designed to mimic that of a real UE. During the measurement, two Loon HAPs were present at $\sim 40 \mathrm{~km}$ and $\sim 25 \mathrm{~km}$ from the test location and data was collected from both simultaneously. Channel impulse response data was captured for $1 \mathrm{~h}$ while keeping the TSME antenna stationary using the LTE pilot signals from both Loon HAPs. Measurements were taken on the ground floor of a single story building for the indoor results.

Figure 12 shows the signal power distribution from the HAP that is $\sim 40 \mathrm{~km}$ (i.e., elevation angle of $\sim 25^{\circ}$ from the test location towards the HAP). Both indoor and outdoor measurements are shown. The difference in mean between outdoor and indoor is $7 \mathrm{~dB}$ with a standard deviation of $4.6 \mathrm{~dB}$ indoors and $4.7 \mathrm{~dB}$ outdoors. Shimamoto et al. [17] measures a standard deviation of $2.65 \mathrm{~dB}$ at $20^{\circ}$, $1.75 \mathrm{~dB}$ at $30^{\circ}$, and $3.9 \mathrm{~dB}$ at $40^{\circ}$. Measurements from the Loon HAP are slightly higher than this at this location.

However, a higher standard deviation is expected indoors, indicating that there is not a significant amount of multi-path. More testing in indoor locations is required to further understand this observation.

Figure 13 shows the same plot from the HAP that is $\sim 25 \mathrm{~km}$ away from the test location (i.e., elevation angle of $\sim 45^{\circ}$ from the test location towards the HAP). In this case, the difference in mean is $6.7 \mathrm{~dB}$ with a standard deviation of $2.5 \mathrm{~dB}$ indoors and $3.5 \mathrm{~dB}$ outdoors. This roughly matches the measurement of $3.9 \mathrm{~dB}$ at $40^{\circ}$ from [17].

\subsubsection{Diversity/MIMO communication}

Using KPI data collected from the Loon network in Peru and Puerto Rico, on average, rank-2 communication is realized less than $10 \%$ of the time. This is expected due to the prior discussion on UE antenna construction in bands lower than $1 \mathrm{GHz}$. Figure 14 shows the CDF

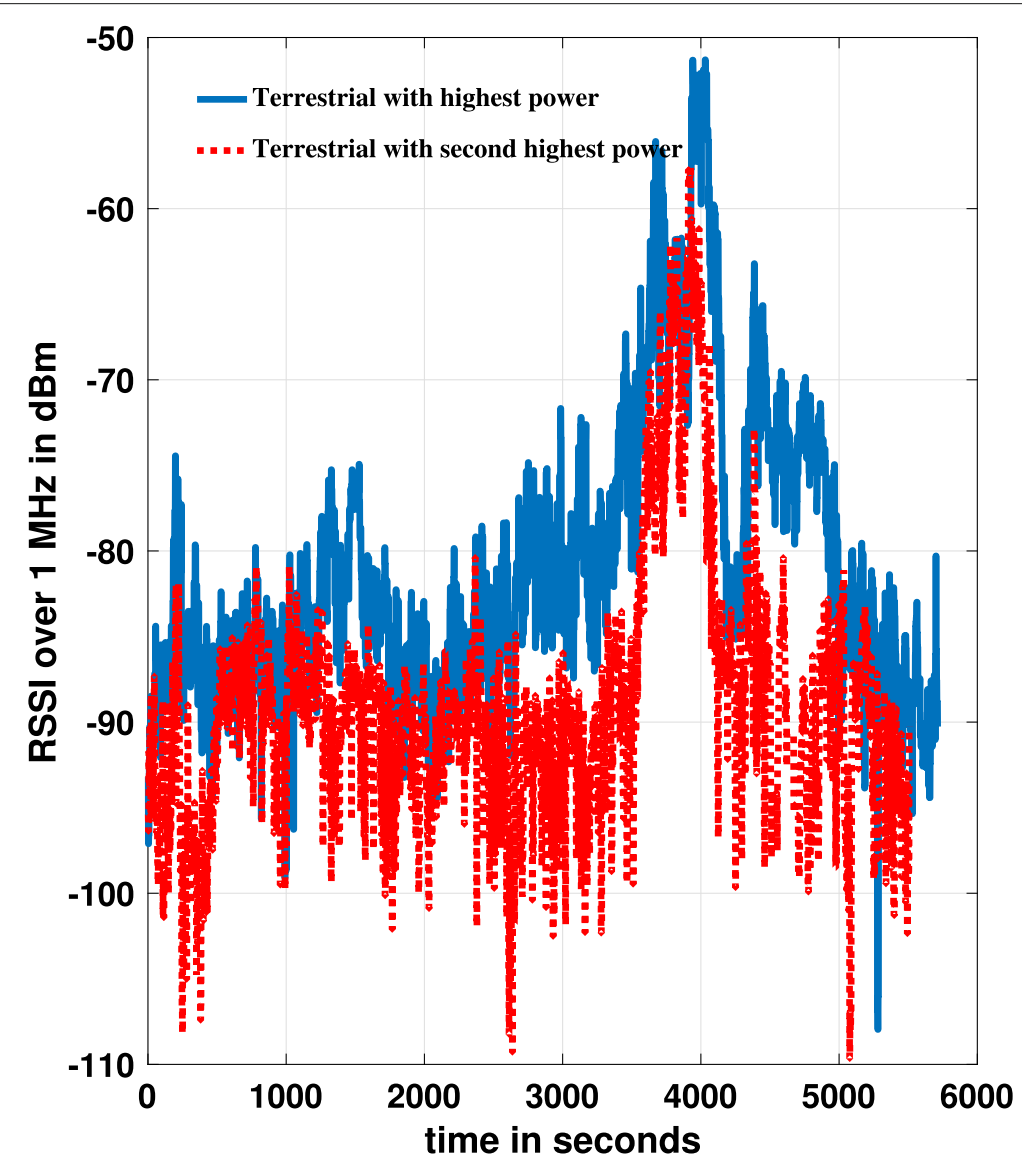

Fig. 16 RSSI from two terrestrial towers 
of polarization diversity by taking the ratio of rank-2 requests to the sum of rank-1 and rank-2 requests from KPI data.

An experimental configuration where the Loon eNB employed spatial diversity instead of polarization diversity was also tested. In this experiment, the transmit antennas were placed $\sim 1 \mathrm{~m}$ apart with the same polarization. From the theory presented in [12], the antennas should have been spaced further apart. However, the prototype Loon platform limitations restricted the spacing that could be achieved. Figure 14 also shows the CDF of this experiment. It can be seen that polarization diversity is significantly better than this particular configuration of spatial diversity. In addition, neither diversity scheme is sufficient for MIMO communication to a large number of UEs. It is expected that improving antenna gain and tweaking the ECC at the eNB will improve this situation. However, even with this improvement, we expect the MIMO performance from a HAP platform at lower frequencies to be worse than what is seen in a terrestrial network.

\subsection{Terrestrial coexistence}

\subsubsection{DL interference-terrestrial only}

An analysis of DL terrestrial self interference was performed against a live terrestrial network. For this analysis, the TSME equipment was used to take measurements in a vehicle as part of a limited drive test of the terrestrial network. The SINR from this measurement is presented in Fig. 15 and the Received Signal Strength Indicator (RSSI) from the same measurement is presented in Fig. 16.

As can be seen, the RSSI from both towers is quite high for the duration of the test and especially around $4000 \mathrm{~s}$. However, since the two towers have similar RSSI, the interference resulted in an SINR that is much lower than expected. In this case, the average SINR is on the order of $10 \mathrm{~dB}$ with substantial dips around $4000 \mathrm{~s}$ when both terrestrial towers have elevated RSSI.

\subsubsection{DL interference-terrestrial with a Loon HAP}

Several measurement campaigns were conducted in Piura, Peru against a live, terrestrial partner network. During the campaigns, SINR was systematically measured on the ground using the TSME equipment at various distances from a terrestrial tower. A tower close to the edge of a terrestrial network's coverage area was chosen for this campaign. A Loon eNB was then turned on and off at known time intervals to assess the impact on SINR. During all measurements, no degradation of the terrestrial tower was observed.

Assessment of KPIs from the partner network in the Piura, Peru region were also observed. For this

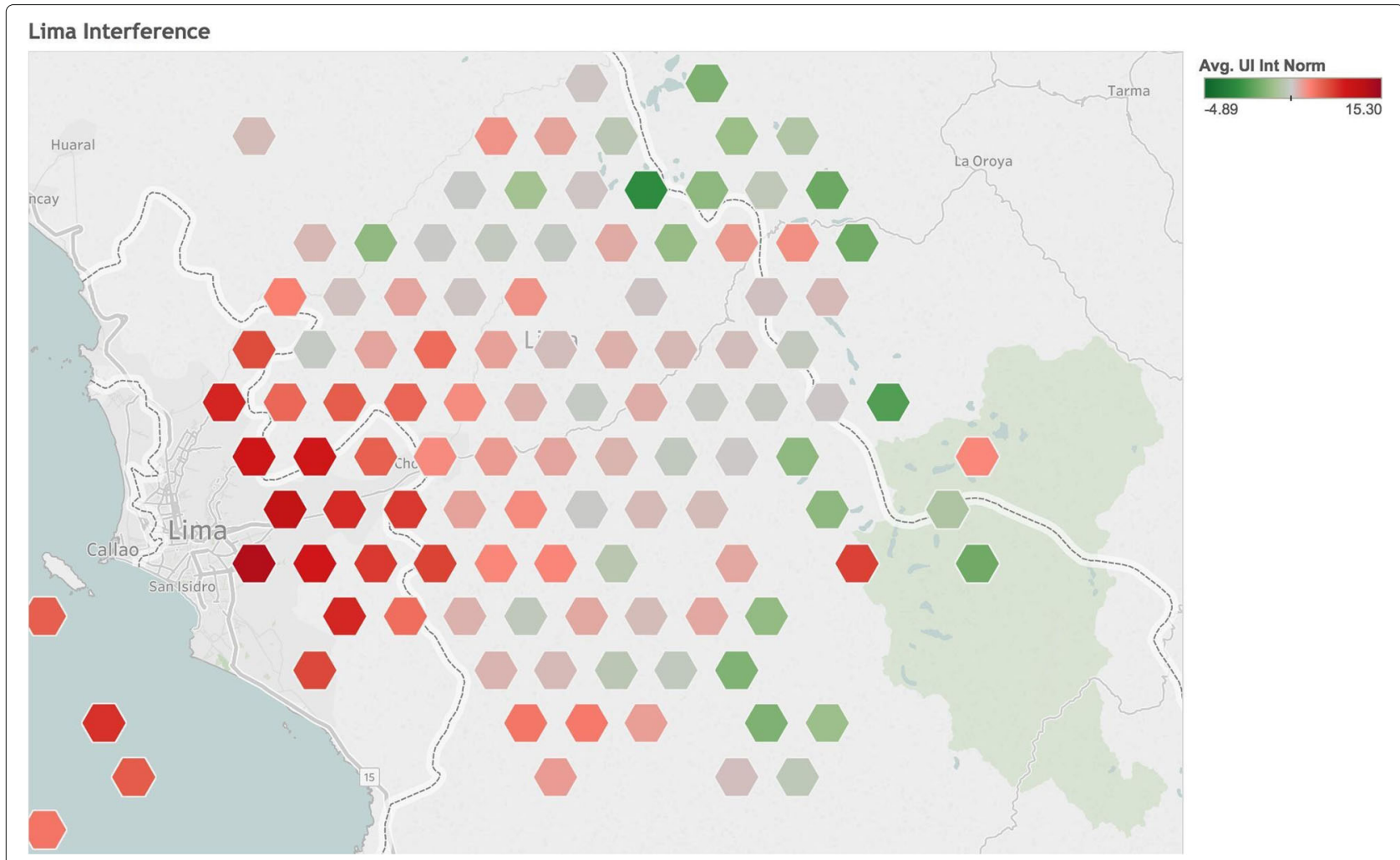

Fig. $17 \cup L$ Interference observed from Loon near Lima 
assessment, DL and UL throughput and PRB utilization KPIs were collected over the course of a month. During this time, the Loon network was not operating at all times and the times of operation were recorded. An analysis was then conducted over all the KPIs to determine the delta between when the Loon network was present and not present. This analysis showed that no degradation was observable in the partner network's KPIs.

\subsubsection{UL interference}

KPIs from the Loon network were collected over the course of several months over Lima, Peru to analyze the effect of UL interference. For this analysis, UL noise KPIs were averaged over all Loon HAPs using a geographic grid with $11 \mathrm{~km}$ spacing. These noise measurements are not calibrated and can therefore only be used for a relative assessment and not for an absolute assessment.

Figure 17 shows the collected UL noise data as a heatmap. As can be seen, the delta in UL noise is $20.1 \mathrm{~dB}$ (a range of -4.8 to $15.3 \mathrm{~dB}$ is observed) with the highest interference being closest to Lima, where the terrestrial network was the most dense.

\section{Conclusion}

This paper describes physical layer aspects involved in the Loon system design. Specifically, the following has been shown:

- Coexistance of terrestrial and HAP LTE networks: With careful system design it is possible for a HAP LTE network to coexist with terrestrial LTE networks and that the addition of a Loon HAP has a minor effect on DL interference, which is offset by a substantial increase in coverage.

- MIMO challenges below $1 \mathrm{GHz}$ : At frequencies below $1 \mathrm{GHz}$ utilizing polarization diversity to achieve MIMO links has challenges due to UE antenna pattern limitations. These limitations are more pronounced as the UE becomes smaller.

- UE energy consumption when used with HAP LTE networks: Increase in energy consumption in the UE while communicating with HAPs is not significant in normal use cases (i.e., less than $1 \%$ of UE battery life).

\begin{abstract}
Abbreviations
AWGN: Additive White Gaussian Noise; BPL: Building penetration loss; CDF: Cumulative density function; CDMA: Code division multiple access; DL: Downlink; ECC: Envelope correlation coefficient; eNB: eNodeB; elCIC: Enhanced inter cell interference coordination; EVM: Error vector magnitude; FSPL: Free space path loss; HAP: High-altitude platform; ICIC: Inter cell interference coordination; KPI: Key performance indicator; LOS: Line of sight; LTE: Long-Term Evolution; MIMO: Multiple Input Multiple Output; PRB: Physical resource block; RF: Radio frequency; RSSI: Received signal strength indicator; SINR: Signal to interference plus noise ratio; UAV: Unmanned aerial vehicle; UE: User Equipment; UL: Uplink; XPD: Cross Polar Discrimination
\end{abstract}

\section{Acknowledgements}

We would like to thank Dr Behrouz Babakhani and Mr John Shamblin for useful discussion around the topic of ECC.

\section{Funding}

Not applicable.

\section{Availability of data and materials}

The data sets generated and/or analyzed during the current study are not publicly available as they have been collected on private partner networks.

\section{Authors' contributions}

SA carried out the simulation studies, validated data against simulation, and codrafted the manuscript. BW lead the measurement campaign and co-drafted the manuscript. $N G, A C, A B$, and $B F$ helped in the measurement campaign and data analysis. All authors read and approved the final manuscript.

\section{Competing interests}

The authors declare that they have no competing interests.

\section{Publisher's Note}

Springer Nature remains neutral with regard to jurisdictional claims in published maps and institutional affiliations.

Received: 18 December 2018 Accepted: 30 April 2019

Published online: 25 June 2019

\section{References}

1. F. Dovis, R. Fantini, M. Mondin, P. Savi, Small-scale fading for high-altitude platform (hap) propagation channels. IEEE J. Sel. Commun. 20(3), 641-647 (2002)

2. F. Dovis, R. Fantini, M. Mondin, P. Savi, in Global Telecommunications Conference, 2001. GLOBECOM'01. IEEE, vol. 1. $4 \mathrm{~g}$ communications based on high altitude stratospheric platforms: Channel modeling and performance evaluation (IEEE, 2001), pp. 557-561

3. S. Zvanovec, P. Piksa, M. Mazanek, P. Pechac, A study of gas and rain propagation effects at $48 \mathrm{ghz}$ for hap scenarios. EURASIP J. Wirel. Commun. Netw. 2008, 19 (2008)

4. M. Vázquez-Castro, F. Pérez-Fontán, B. Arbesser-Rastburg, Channel modeling for satellite and haps system design. Wirel. Commun. Mob. Comput. 2(3), 285-300 (2002)

5. M. Kvicera, P. Pechac, Building penetration loss for satellite services at I-, s-and c-band: measurement and modeling. IEEE Trans. Antennas Propag. 59(8), 3013-3021 (2011)

6. J. Holis, P. Pechac, Penetration loss measurement and modeling for hap mobile systems in urban environment. EURASIP J. Wirel. Commun. Netw. 2008, 18 (2008)

7. J. Holis, P. Pechac, Elevation dependent shadowing model for mobile communications via high altitude platforms in built-up areas. IEEE Trans. Antennas Propag. 56(4), 1078-1084 (2008)

8. D. I. Axiotis, M. E. Theologou, in Global Telecommunications Conference, 2002. GLOBECOM'02. IEEE, vol. 2. 2 ghz outdoor to indoor propagation at high elevation angles for mobile communications served by high altitude platform stations (IEEE, 2002), pp. 1865-1869

9. M. Kvicera, P. Pechac, Influence of antenna characteristics on elevation dependence of building penetration loss for high elevation links. Radioengineering. 21(4), 1031 (2012)

10. C. Oestges, in Vehicular Technology Conference, 2008. VTC Spring 2008. IEEE. Impact of tropospheric depolarization on the performance of dual-polarized high-altitude platform systems (IEEE, 2008), pp. 2897-2901

11. A. G. Kanatas, A. D. Panagopoulos, Radio Wave Propagation and Channel Modeling for Earth-Space Systems. (CRC Press, 2016)

12. E. T. Michailidis, A. G. Kanatas, Three-dimensional HAP-MIMO channels: modeling and analysis of space-time correlation. IEEE Trans. Veh. Technol. 59(5), 2232-2242 (2010)

13. K. Siahaan, et al, in Telecommunication Systems Services and Applications (TSSA), 2016 10th International Conference On. Performance improvement on the downlink haps communication channel employing mimo antenna (IEEE, 2016), pp. 1-4

14. J. L. Cuevas-Ruiz, A. Aragon-Zavala, M. A. Lopez-Consospo, in Electrical, Communications, and Computers, 2009. CONIELECOMP 2009. International Conference On. Mimo channels: an approach for haps communication systems (IEEE, 2009), pp. 130-133

15. F. Dong, M. Li, X. Gong, H. Li, F. Gao, Diversity performance analysis on multiple hap networks. Sensors. 15(7), 15398-15418 (2015) 
16. V. Nikolaidis, N. Moraitis, A. G. Kanatas, Dual-polarized narrowband MIMO LMS channel measurements in urban environments. IEEE Trans. Antennas Propag. 65(2), 763-774 (2017)

17. S. Shimamoto, et al., Channel characterization and performance evaluation of mobile communication employing stratospheric platforms. IEICE Trans. Commun. 89(3), 937-944 (2006)

18. E. T. Michailidis, P. Petropoulou, A. G. Kanatas, in 2013 7th European Conference on Antennas and Propagation (EUCAP). Geometry-based modeling of cross-polarization discrimination in hap propagation channels (IEEE, 2013), pp. 2296-2300

19. A. M. Turkmani, A. A. Arowojolu, P. Jefford, C. Kellett, An experimental evaluation of the performance of two-branch space and polarization diversity schemes at 1800 mhz. IEEE Trans. Veh. Technol. 44(2), 318-326 (1995)

20. R. G. Vaughan, Polarization diversity in mobile communications. IEEE Trans. Veh. Technol. 39(3), 177-186 (1990)

21. S. Sirianunpiboon, S. Howard, A. Calderbank, in Information Theory for Wireless Networks, 2007 IEEE Information Theory Workshop On. Diversity gains across line of sight and rich scattering environments from space-polarization-time codes (IEEE, 2007), pp. 1-5

22. F. Boccardi, B. Clerckx, A. Ghosh, E. Hardouin, G. Jöngren, K. Kusume, E. Onggosanusi, Y. Tang, Multiple-antenna techniques in Ite-advanced. IEEE Commun. Mag. 50(3) (2012)

23. J. HOLIŠ, P. Pechac, Coexistence of terrestrial and HAP $3 G$ networks during disaster scenarios. Radioengineering. 17(4) (2008)

24. P. Likitthanasate, D. Grace, P. Mitchell, Coexistence performance of high altitude platform and terrestrial systems sharing a common downlink wimax frequency band. Electron. Lett. 41(15), 858-860 (2005)

25. D. Grace, J. Thornton, G. Chen, G. P. White, T. C. Tozer, Improving the system capacity of broadband services using multiple high-altitude platforms. IEEE Trans. Wirel. Commun. 4(2), 700-709 (2005)

26. J.-M. Park, D.-S. Oh, Y.-S. Kim, D.-S. Ahn, in Global Telecommunications Conference, 2003. GLOBECOM'03. IEEE, vol. 1. Evaluation of interference effect into cellular system from high altitude platform station to provide imt-2000 service (IEEE, 2003), pp. 420-424

27. J.-M. Park, B.-J. Ku, Y.-S. Kim, D.-S. Ahn, in Wireless Personal Multimedia Communications, 2002. The 5th International Symposium On, vol. 3. Interference analysis of cellular system due to haps system to provide imt-2000 service (IEEE, 2002), pp. 1182-1186

28. A. Widiawan, R. Tafazolli, Analytical investigation on sharing band overlaid high altitude platform station-terrestrial cdma system. Electron. Lett. 41(2), 77-79 (2005)

29. TR36.824, Lte coverage enhancements (2012). http://www.qtc.jp/3GPP/ Specs/36824-b00.pdf. Accessed 31 Mar 2019

30. B. Mun, F. J. Harackiewicz, B. Kim, H. Wi, J. Lee, M.-J. Park, C. Jung, B. Lee, New configuration of handset MIMO antenna for LTE 700 band applications. Int. J. Antennas Propag. 2013 (2013). https://www.hindawi. com/journals/ijap/2013/850489/abs/

31. A. Derneryd, J. Fridén, A. Stjernman, in 2010 URSI International Symposium on Electromagnetic Theory. Mimo performance of closely spaced antennas in the $700 \mathrm{mhz}$ band (IEEE, 2010), pp. 219-222

32. B. Hagerman, K. Werner, J. Yang, in 2011 IEEE Vehicular Technology Conference (VTC Fall). Mimo performance at $700 \mathrm{mhz}$ : Field trials of Ite with handheld ue (IEEE, 2011), pp. 1-5

33. A. Elnashar, M. A. El-saidny, M. R. Sherif, Coverage and capacity planning of $4 \mathrm{G}$ networks. Design, Deployment and Performance of 4G-LTE Networks: A Practical Approach, 349-444 (2014)

34. J. Holis, D. Grace, P. Pechac, Effect of antenna power roll-off on the performance of $3 \mathrm{G}$ cellular systems from high altitude platforms. IEEE Trans. Aerosp. Electron. Syst. 46(3), 1468-1477 (2010)

35. I. Recommendation, 1456:'minimum performance characteristics and operational conditions for high altitude platform stations providing imt-2000 in the bands 1885-1980 mhz, 2010-2025 mhz and 2110-2170 $\mathrm{mhz}$ in the regions 1 and 3 and 1885-1980mhz and 2110-2160mhz in region 2. International Telecommunications Union (2000)

36. TR25.816, UMTS 900 MHz Work Item Technical Report (2005-2012). http:// www.qtc.jp/3GPP/Specs/25816-800.pdf. Accessed 31 Mar 2019

37. E. U. T. R. Access, Base station (bs) radio transmission and reception", 3gpp ts 36.104 versin 10.1. 0 release 10. Technical Specification (2011)

38. P. Joshi, D. Colombi, B. Thors, L.-E. Larsson, C. Törnevik, Output power levels of $4 \mathrm{~g}$ user equipment and implications on realistic rf emf exposure assessments. IEEE Access. 5, 4545-4550 (2017)
39. B. Dusza, C. Ide, L. Cheng, C. Wietfeld, in Computer Communications Workshops (INFOCOM WKSHPS), 2013 IEEE Conference On. An accurate measurement-based power consumption model for Ite uplink transmissions (IEEE, 2013), pp. 49-50

40. J. Wigard, T. Kolding, L. Dalsgaard, C. Coletti, in Communications Workshops, 2009. ICC Workshops 2009. IEEE International Conference On. On the user performance of Ite ue power savings schemes with discontinuous reception in Ite (IEEE, 2009), pp. 1-5

41. Tsme ultacompact drive test scanner (2016). https://cdn.rohdeschwarz. com/pws/dl_downloads/dl_common_library/dl_manuals/gb_1/t/ tsme_1/TSME_UserManual_en_10.pdf

\section{Submit your manuscript to a SpringerOpen ${ }^{\circ}$ journal and benefit from:}

- Convenient online submission

- Rigorous peer review

- Open access: articles freely available online

- High visibility within the field

- Retaining the copyright to your article

Submit your next manuscript at $\boldsymbol{s p r i n g e r o p e n . c o m ~}$ 